\title{
Privatización de la educación y poder corporativo: El fenómeno en una comuna urbano-popular en Santiago de Chile
}

\author{
Privatization of education and corporate power: The phenomenon in an urban \\ low income neighborhood in Santiago of Chile
}
A privatização da educação e do poder corporativo: O fenômeno em um bairro urbano popular em Santiago de Chile

\author{
Diego Parra Moreno' \\ Juan Gonzaléz López" \\ 'Universidad de Chile -(UCHILE), Santiago de Chile - email dparram@ug.uchile.cl \\ "Universidad de Chile -(UCHILE), Santiago de Chile - email juangl@uchile.cl
}

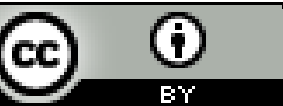

Educação: teoria e prática, Rio Claro, SP, Brasil - eISSN: 1981-8106

Está licenciada sob Licença Creative Common

\section{Resumen}

El presente artículo expone los resultados de un estudio de caso sobre el fenómeno de la privatización de la educación obligatoria en Chile. Se analizan datos y redes empresariales y religiosas de los principales sostenedores privados o Grupos de Poder ubicados en la comuna urbano-popular de La Pintana, Región Metropolitana, entre el año 1995 al 2014. Los resultados indican el crecimiento y consolidación de los sostenedores privados, como también de las Alianzas Público-privadas y vínculos con empresas nacionales y transnacionales. El estudio señala la creciente influencia de los sostenedores privados y sus redes en el sistema educativo a través de diversos procesos de privatización.

Palabras-clave: Privatización de la Educación. Sostenedores Privados. Poder Corporativo. Redes empresariales y religiosas.

\footnotetext{
Abstract

This paper presents the results of a case study on the phenomenon of privatization of compulsory education in Chile. Data from the largest education providers (stakeholders) or Groups of Power from the urban low income neighborhood of La Pintana is revised. The data includes corporate/business and religious networks of these stake holders between the period 1995 to 2014. There search finding sindicate a growth and consolidation of these education providers over the years. Further, there is also evidence of increase of both public-private partnerships as well as links to national and transnational corporations. The study highlights the steady growing influence of these organization sand their networks in the educational system through diverse processes of privatization.
} 
Keywords: Privatization of Education. Education providers (stakeholders). Corporate Power. Corporate/business and religious networks.

\section{Resumo}

Este artigo apresenta os resultados de um estudo de caso sobre o fenômeno da privatização relativo à escolaridade obrigatória no Chile. Dados são analisados, bem como as redes empresariais e religiosas que compõem os Grupos de Poder, ou seja, os maiores prestadores de ensino privado localizadosnum bairro urbano popular, La Pintana, Región Metropolitana, entre 1995 a 2014. Os resultados indicam o crescimento e consolidação dos prestadores de serviços educacionais, de natureza privada, bem como das parcerias público-privadas e ligações com empresas nacionais e transnacionais. $O$ estudo enfatiza a crescente influência dos prestadores de ensino privado e de suas redes no sistema de ensino por meio de diferente processos do privatização.

Palavras-chave: Privatização da educação.Prestadores de ensino privado. Poder Corporativo. Redes empresariais e religiosas.

\section{La Privatización de la Educación en el Chile Neoliberal}

El sistema educativo chileno ha sido considerado un experimento neoliberal a nivel económico, social y cultural-ideológico (REDONDO, 2005; CORNEJO, 2006; ASSAÉL et al., 2011). La reforma neoliberalha reconfigurado la institucionalidad,el financiamientoy el sentido de la escuela pública en Chile.En dictadura,las escuelas se traspasaron administrativamente a los municipios y a privados, que actuarían como proveedores educativos, implementándose un modelo de financiamientobasado en un subsidio a la oferta un vouchers a la chilena- el cual consiste en una subvención porel promedio de asistencia mensual de cada estudiante.

Esta subvención se entrega directamente a los sostenedores, quienes pagan al personal docente y todos los gastos de las escuelas, administrándolas totalmente.Esto desvinculó definitivamente al Estado de las escuelas, dando todo el poder a los sostenedores.En el año 1993,se aprobó la posibilidad de que los proveedores privados pudiesen cobrar un monto adicional a las familias, el Financiamiento Compartido $\left(\mathrm{FICOM}^{1}\right)$, lo que permitió la consolidación de la oferta privada, aumentando su cobertura.En treinta años de competencia,entre la oferta educativa privada y municipal,se ha generado un cambio en la subjetividad de la población chilena. La educación estatal es sinónimo de precarización, lo

\footnotetext{
${ }^{1}$ Financiamiento compartido, medida instaurada enchile el año 1994, que permite a los sostenedores hacer un cobro en dinero a las familias. Esta medida ha contribuido significativamente a la segregación del sistema educativo.

Educação: Teoria e Prática/ Rio Claro/ Vol. 25, n.50/ p. XXX-XXX/ Set.-Dez. 2015.
} 
privado aparece como única alternativa para las familias que desean educación de calidad, sin importar quién sea el proveedor, o lo que implique tal promesa. Inclusive, algunos estudios han evidenciado que la educación privada no ha generado mejor calidad en el sistema educativo (REDONDO et al., 2004; BELLEI, 2007).

En consecuencia, cada vez más escuelas y liceos pasan a ser controlados por agentes privados.¿Quiénes son estos agentes? ¿Son organizaciones sociales? ¿Religiosas? ¿Asociaciones docentes? ¿De padres? Existe poco conocimiento sobre quiénes son los proveedores de la educación particular subvencionada en Chile (ALMONACID, 2008). Conocer quiénes son los principales proveedores privados es fundamental para conocer la diversidad u homogeneidad del sistema. El control de los sistemas educativos por los grupos de poder económico es una tendencia en el mundo y en Latinoamérica (BALL, 2012; CLADE, 2015).

\section{Gobernanza y Educación}

En los estudios de privatización, que comienzan a caracterizar este fenómeno, el problema de la gobernanza en relación a los sistemas educativos es un tema fundamental. A pesar que el foco parece estar, únicamente, en la calidad educativa, existen trabajos que muestran cómo los grupos de poder económico, gracias a la creciente ola de privatización, se hacen del control de los sistemas educativos a nivel local, nacional y/o trasnacional.

Por ejemplo, Stephen Ball(2014) ve en la privatización educativa una herramienta para constituir nuevas condiciones para la gobernabilidad en sociedades cada vez más complejas, argumentando que la presencia del sector privado se debe entender en un nuevo contexto de las políticas educativas, las cuales pasan de un sistema basado en el control burocrático a un sistema basado en contratos, de ventas de políticas educativas, como un medio para administrar resultados en vez de procesos.

La contratación y descentralización de estos servicios introduce, además, nuevos tipos de actores al ámbito de la función Estatal, ampliando los espacios y modos de gobernar, introduciendo nuevos tipos de relaciones de poder. Este nuevo tipo de gobernanza educativa se articularía en "redes auto organizadas e inter organizacionales caracterizadas por la interdependencia, intercambio de recursos, reglas del juego y autonomía significativa con respecto al Estado" (RHODES, 1997, p. 15 citado en BALL; CORBALÁN, 2013).De este modo, los servicios públicos ensayan una composición de "alianzas estratégicas, acuerdos de Educação: Teoria e Prática/ Rio Claro/ Vol. 25, n.50/ p. XXX-XXX/ Set.-Dez. 2015. 
trabajo conjuntos, redes, asociaciones y muchas otras formas de colaboración que traspasan las fronteras sectoriales y organizacionales" (WILLIAMS, 2002, p. 103 citado en BALL; CORBALÁN, 2013).

Otro elemento que caracteriza a este tipo de actores, que interviene en la educación pública, es el descrito por Toni Verger (2013),acerca de las llamadas PublicPrivatePartnership(PPP's) o Alianzas Público-Privada,entre este tipo de actores empresariales y el Estado. Los PPPs se definen como una relación contractual entre el sector público y el sector privado, donde el sector privado se compromete a proveer un servicio, en este caso educativo, a un determinado precio y por un determinado tiempo. Esta política, impulsada por el Banco Mundial, pretende, entre otros objetivos, redireccionar fondos destinados al gasto público, hacia los privados y, así, fortalecer la alternativa privada en educación, generar más eficiencia en el manejo de recursos y debilitar los sindicatos docentes, entre otros beneficios para la estrategia financiera nacional y/o transnacional (VERGER, 2013).

La Campaña Latinoamericana por el Derecho a la Educación (CLADE), ilustra sobre este fenómeno en toda Latinoamérica. El cual describen como un fenómeno complejo, que llaman "la gobernanza corporativa" y se caracteriza por:

[...] la participación directa de representantes de corporaciones en la definición de la agenda de la educación pública, a través de mecanismos que no consultan los espacios de participación social puestos en marcha para la deliberación y la toma democrática de decisiones sobre la política pública(CLADE, 2015; p.21).

Esto lo hacen mediante la constitución de redes de empresarios que buscan influir en la legislación y provisión educativa, mediante programas educativos financiados por empresas privadas o a través de la influencia directa de representantes de este sector en la toma de decisiones sobre educación (CLADE, 2015).

Por lo tanto, los grupos de poder presentes en la provisión educativa son un fenómeno al que hay que prestar especial atención en el complejo fenómeno de la privatización educativa. Sus proyectos educativos, la matrícula que gestionan, su relación con el Estado y las redes entre ellos, deben ser visualizadas con el objetivo de caracterizar mejor nuestros sistemas educativos, como complejos políticos de influencia social, más allá de las ganancias que los proveedores educativos pueden obtener de los recursos públicos destinados a la 
educación pública, que, si bien es un ámbito de preocupación, no debe ser el único al cual debe prestarse atención desde la perspectiva de promover sistemas educativos democráticos.

\section{Grupos de Poder en Chile}

Entre el año 2009 y 2010 se desarrolló una caracterización de los proveedores privados de la educación particular subvencionada (sostenedores) en la Región Metropolitana de Chile, considerando que es la región con la mayor presencia de este sector en el país. Revisamos las redes comerciales, políticas e ideológicas de una muestra extraída aleatoriamente de las personas -naturales o jurídicas ${ }^{2}$ - que sostenían directamente escuelas particulares subvencionadas, buscando realizar una primera caracterización del sector, diferenciándolos así entre ellos por sus relaciones económicas, políticas y su influencia en el sector educativo (CORBALÁN; LIGÜEÑO; GONZÁLEZ, 2009; GONZÁLEZ et al., 2010; GONZÁLEZ; LIGÜEÑO; PARRA, 2012).

En el mapeo realizado se evidenció, fundamentalmente,cuatro tipos de proveedores. Los primeros, muy numerosos, los llamamos asalariados, sin relaciones económicas y políticas significativas, dado que poseían una sola escuela y vivían exclusivamente de su trabajo como proveedores educativos, en base a lo que lograban extraer como ganancia de la administración de los recursos estatales entregados para gestionar las escuelas. Los segundos, también muy numerosos, los llamamos comerciantes, asociados a uno o dos colegios, aparecían vinculados a otras actividades comerciales, o productivas, no siendo la gestión escolar su única actividad. Ambos grupos, en general, tienen escuelas pequeñas de menos 1000 estudiantes. En tercer lugar, aparecen los que llamamos inversionistas, personas naturales y/o jurídicas, que se vinculan directamente o indirectamente a dos o más establecimientos escolares, y que, además, aparecían en otras actividades comerciales, industriales o financieras, siendo miembros de sociedades y/o directorios de empresas, miembros de sociedades anónimas, o accionistas, resultando la escuela una actividad más entre otros rubros de negocio más importantes, lo que les permite tener capacidad financiera para invertir, construyendo escuelas de más de 1000 estudiantes y logrando mejores ganancias de la actividad educativa.

\footnotetext{
${ }^{2}$ Estos sostenedores se identifican a través de una figura jurídica (Sociedad Anónima, Sociedad Limitada, Fundaciones, Corporaciones y Empresa Individual Responsabilidad Limitada E.I.R.L.) o persona naturales. Según el artículo transitorio $\mathrm{N}^{\circ} 1$ de la Ley General de Educación (2009) señala que los sostenedores que figuran como persona natural tenían un plazo de dos años para cambiar a persona jurídica, situación que a la fecha no se cumple.
} 
Todos estos grupos se constituyen como proveedores entorno al lucro, es decir, a la menor o mayor extracción de ganancias que pueden hacer de la gestión que hacen de la subvención que les entrega el Estado y/o el pago que hacen las familias para la educación de sus hijos/as. Organizaciones sociales de base, colectivos docentes, asociaciones de padres, asociaciones eclesiásticas son casi inexistentes en la oferta educativa privada chilena, al menos en la Región Metropolitana, salvo excepciones ${ }^{3}$.

El último grupo de proveedores encontrados, quizás el de mayor importancia política, está constituido por congregaciones católicas e instituciones de beneficencia y filantrópicas, estrechamente ligadas a los grupos de poder económico del país. Se vinculan en redes y logran incidir en la toma de decisiones sobre la educación pública. Estos sostenedores educativos participan en directorios de empresas controladoras de recursos naturales, industriales y financieros, se vinculan a sectores conservadores de la Iglesia Católica y mantienen diversas relaciones entre sí. Por esto los hemos llamado el Complejo ReligiosoEmpresarial. ${ }^{4}$ Hipotetizamos que, en este complejo de proveedores, no existe una motivación vinculada al lucro, sino un interés, más bien, ideológico. Este afán ha estado presente hace más de un siglo en la iglesia y en grupos de nuestra oligarquía nacional, que han visto en el control de las escuela la posibilidad de incidir en la gobernanza social (MORRIS, 1967).

\section{Estudio de caso: el Poder Corporativo en una comuna popular en Chile}

5.

6. Para profundizar en la caracterización de la presencia de los grupos de poder privado en la educación financiada por el Estado, hemos decidido realizar un estudio de caso(STAKE, 1998), centrándonos en una comuna urbano-popular (La Pintana, Región Metropolitana). Este estudio consta de un diseño de investigación no experimental de dos fases:

1) Realización de un análisis descriptivo de fuentes primarias y secundarias de información acerca del conjunto de sostenedores particulares subvencionados de la comuna de La Pintana. Estas fuentes corresponden a registros del Ministerio de Educación, archivos del Ministerio de Justicia, registros del Servicios de Impuestos Internos y de la base de datos del

\footnotetext{
${ }^{3}$ Está el caso de la Escuela Casa Azul creada en la comuna de la Granja, que la sostiene profesores y vecinos de la población Yungay, educando cerca de 300 estudiantes. Otro ejemplo, es la Escuela Raimapu en la comuna de La Florida, la cual es administrada por apoderados, ex apoderados y trabajadores de la escuela, donde asisten aproximadamente 800 estudiantes.

${ }^{4}$ Ver más sobre este tema

Educação: Teoria e Prática/ Rio Claro/ Vol. 25, n.50/ p. XXX-XXX/ Set.-Dez. 2015.
} 
Diario Oficial. Esto nos permitió establecer las redes políticas y económicas del sostenedor, permitiéndonos clasificarlo dentro de las categorías ya establecidas en un estudio anterior.

2) En base a esto, identificamos a los proveedores educativos subvencionados asociados a los grupos de poder económico y religioso, revisando la concentración de matrículas y número de establecimientos entre el año 1995 y 2014. Además, incorporamos variables asociadas a las escuelas tales como: existencia o ausencia de FICOM y puntaje $\operatorname{IVE}^{5}$ de cada establecimiento.

\section{1. ¿Por qué en la comuna de La Pintana?}

\section{2 .}

La comuna de La Pintana, ubicada en el sector sur del Gran Santiago ${ }^{6}$, se crea el año 1984, en plena dictadura militar, territorio de origen rural y organización feudal (haciendas). Cuenta con 182.930 habitantes (CHILE/INE, 2012) y según el Índice de Calidad de Vida Urbana (IEU, 2014) ${ }^{7}$ presenta un uno de los peores puntajes entre las 93 ciudades de todo Chile(36,1), ubicándose muy por debajo del promedio nacional $(46,1)$. La municipalidad, por su parte, reconoce que su pobreza es "extraordinariamente homogénea" (SECRETARÍA DE PLANIFICACIÓN COMUNAL, 2012).

Respecto a la provisión educativa al 2014, existen 56 sostenedores a cargo de 72 establecimientos educacionales con subsidio estatal (escuelas, liceos y escuelas especiales), atendiendo a una población escolar de 38.440 estudiantes aproximadamente. El sistema educativo municipal administra trece establecimientos que atienden a 6.878 estudiantes $(17,9 \%$ del total), en cambio los 55 sostenedores privados restantes controlan 59 establecimientos educacionales $^{8}$, atendiendo a 31.562 estudiantes (82,1\% del total). En efecto, la tendencia en los últimos 20 años indica un crecimiento progresivo de la oferta privada y

\footnotetext{
${ }^{5}$ El Índice de Vulnerabilidad del Escolar es el porcentaje de estudiantes que se encuentran en condición de vulnerabilidad en cada establecimiento, considerando el nivel socioeconómico de la familia, el nivel de escolaridad de los padres o apoderados y el entorno del establecimiento.

${ }^{6}$ En Chile hay 342 comunas distribuidas en 15 regiones. La Pintana es una de las 52 administraciones locales de la Región Metropolitana.

${ }^{7}$ Estudio realizado por el Instituto de Estudios Urbanos de la Universidad Católica y la Cámara Chilena de la Construcción, considera a las ciudades con una población mayor a 50.000 habitantes y analiza las siguientes dimensiones: condiciones laborales, ambiente de negocios, condiciones socioculturales, conectividad y movilidad, salud y medioambiente, vivienda y entorno.

${ }^{8}$ Existen cuatro sostenedores con 2 establecimientos: Fundación de Educación Nocedal, Sonia Isabel Zapata Diez, Vasco Osvaldo Núñez Herrera y Marcelo Antonio Sanhueza Cisterna Servicio Educacional E.I.R.L.
} 
una mayor captación de población estudiantil (aumento en un 54,7\%), situación contraria a la educación municipal, la cual ha disminuido en un 51\% a partir del año 1995 (Ver Figura1).

Figura1 - Tendencias Matrículas en Establecimientos Educacionales Públicos y Privados, comuna La Pintana (1995-2014)

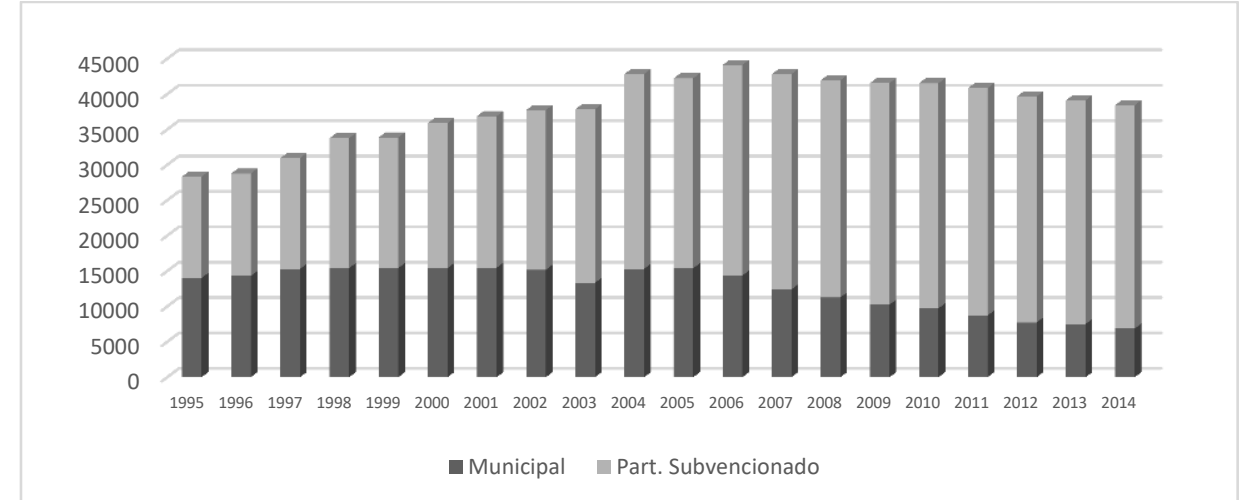

Fuente: Elaboración propia a partir de bases de datos del Ministerio de Educación (MINEDUC)

Otro dato general a considerar en la comuna es el aumento de establecimientos educacionales privados con subvención estatal. Durante las últimas dos décadas, se han creado 43 establecimientos con reconocimiento oficial del Ministerio de Educación, coincidiendo con el inicio del financiamiento compartido en el año 1994 (Figura 2).

Figura 2 - Número de Establecimientos Particulares Subvencionados abiertos cada 10 años, comuna de La Pintana

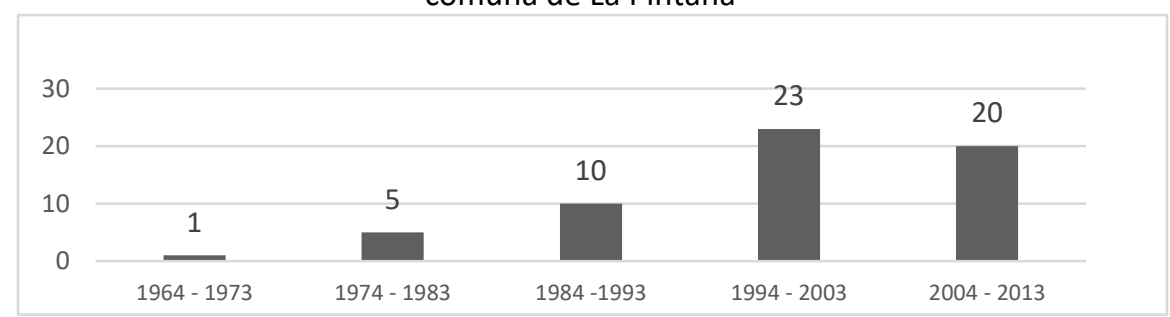

Fuente: Elaboración propia a partir de bases de datos del Ministerio de Educación (MINEDUC)

En síntesis, según los datos la comuna de La Pintana representa un sector de interés para revisar qué tipo de proveedores educativos existen, en especial, la presencia de grupos de poder que pueden estar interviniendo en la gobernanza de una población en riesgo social, que encuentra en la educación privada, financiada por el Estado, la principal alternativa educativa para sus niños/as y jóvenes.

\subsection{Los Grupos de poder en la Comuna urbano-popular de La Pintana}

Cuando hablamos de Grupos de Poder en la educación particular subvencionada nos referimos a los proveedores educativos que tienen vínculos con grupos económicos y/o ideológicos y políticos de influencia en Chile. Estos, mediante fundaciones y corporaciones Educação: Teoria e Prática/ Rio Claro/ Vol. 25, n.50/ p. XXX-XXX/ Set.-Dez. 2015. 
sin fines de lucro, sostienen una o varias escuelas, en general de más de 1000 alumnos, con edificios de buena infraestructura $\mathrm{y}$ buen equipamiento debido al financiamiento complementario que reciben vía donaciones del empresariado ${ }^{9}$. Sus colegios pueden estar en red, conectados entre ellos, o mediante otras fundaciones o instituciones eclesiásticas y/o educativas. De este modo, constituyen redes que coinciden en proyectos educativos, intercambio de recursos, patrocinadores etc. Intervienen en la gestión de establecimientos del Estado y en programas educativos públicos a nivel local y nacional (alianzas públicoprivadas), participan en las mesas técnicas convocadas por las autoridades para la toma de decisiones educativas, ocupan altos cargos ejecutivos y legislativos en el área de la educación pública o están vinculados a instancias de lobby en los procesos de Reforma Educativa.

En la comuna se identificaron nueve sostenedores que se ajustan a la descripción señalada. Estos sostenedores están vinculados a poderosos grupos económicos y/o a la Iglesia católica conservadora, el grupo ideológico con más influencia en Chile. Además presentan diversos niveles de cohesión entre ellos y de influencia en la política pública. Según esto, los podemos dividir en dos subgrupos: a) religiosos y b) empresariales.

a) Grupos religiosos católicos conservadores:son congregaciones, fundaciones y/o corporaciones, ligadas a grupos religiosos o directamente a arzobispados, como el de Santiago. Debido a su vínculo con la Iglesia católica, hacen parte de la poderosa iniciativa de esta entidad en la educación pública, lo que le permite: tener recursos adicionales al financiamiento estatal, vínculos entre ellos y desarrollar iniciativas educativas en distintas comunas y regiones. Según Ball (2012) este tipo de grupos se ampara bajo un mismo principio ideológico, que denomina como la Nueva Filantropía, ya que por medio del capital social acumulado que sostienen en sus redes, logran obtener recursos y legitimidad social por su labor educativa en sectores de pobreza. En su mayoría, son grupos conservadores de la iglesia católica, los cuales comparten un proyecto educativo católico conservador.Por ejemplo, el colegio Nocedal, que se declara laico, señala en su proyecto educativo:

"La única formación doctrinal religiosa que el colegio ofrecerá e impartirá a los padres, profesores, alumnos, personal administrativo y auxiliar, será la católica, con pleno respeto hacia la libertad personal de cada uno. Por otra parte, podrán postular al colegio alumnos de distintos credos religiosos, siempre y cuando sus familias sean respetuosas de la formación católica que

\footnotetext{
${ }^{9}$ Mediante Ley 19.247 de donaciones con fines educacionales, el empresariado puede entregar dinero a estas escuelas, haciendo uso de beneficios tributarios. Por ejemplo, en el bienio 2006-07 de casi 30.000.000 dólares que tuvo el fondo público de donaciones empresariales para la educación, en la Región Metropolitana, el 90\% se lo repartieron entre 55 escuelas ligadas a estos grupos, el resto fue a la educación pública. Así, año tras año este tipo de fondos se concentran en este sector.
} 
aquí se imparte y no pongan obstáculos para que sus hijos asistan regularmente a las clases de Religión y actos de culto, en los que se habrán de comportar de acuerdo a lo que señalen las normas litúrgicas." (COLEGIO NOCEDAL, $2013^{10}$ )

En el caso de La Pintana, contamos entre estos grupos a la Fundación San Marcelino Champagnat, Corporación Educacional Arzobispado de Santiago, Corporación Belén Educa, la Fundación Padre Álvaro Lavín, Fundación Educacional Didascalio y la Fundación Educación Nocedal.

La Fundación Marcelino Champagnat adquirió su personalidad jurídica privada mediante decreto del Obispado de San Bernardo, firmado por el Obispo de dicha diócesis, Monseñor Orozimbo Fuenzalida, y se vincula a la red de establecimientos Maristas, que tiene bajo diversas figuras doce escuelas en distintas comunas del país. Otras Fundaciones ligadas al mismo Obispado, son la Fundación Educación Nocedal, la cual posee tres colegios y extensas redes en el mundo empresarial. Su dirección espiritual está a cargo de la Prelatura del Opus Dei. Esta Fundación ha recibido cuantiosas donaciones del empresariado vía Ley 19.247. Por ejemplo, en el bieno 2008-2009 recibió más de 226 millones de pesos bajo este resquicio (US 361.000 aprox.), además de poseer relaciones de colaboración con empresas que actúan como donantes y/o patrocinadores. Un tercer sostenedor bajo este Obispado es la Fundación Educacional Didascalio, ligada a la Unión Lumen Dei, asociación privada de fieles de la Iglesia Católica. A través de la vicaría de la Educación de San Bernardo, se vincula con quince escuelas en seis comunas correspondientes al sector sur del Gran Santiago (San Bernardo, Calera de Tango, Paine, Pirque, El Bosque, La Pintana).

Otros sostenedores pertenecen a la Arquidiócesis de Santiago, como la Corporación de Educación del Arzobispado de Santiago, con 10 colegios en distintas comunas de la Región Metropolitana y la Fundación Educación Belén, con doce colegios en diferentes comunas del país. Esta última, en el bienio 2008-2009, recibió casi el 50\% de todas las donaciones empresariales vía ley, es decir 1.527 millones de pesos (US 2.443 .000 aprox.). Entre los representantes de su directorio se encuentra como presidente el Arzobispo de Santiago, Monseñor Ricardo Ezzati, y vice-presidente un ex Ministro de Educación del gobierno de Eduardo Frei Ruiz-Tagle (1994-2000), José Pablo Arellano. De las mencionadas, ambas dependen directamente del Arzobispado de Santiago(Figura 3).

\footnotetext{
${ }^{10}$ Disponible en: http://www.colegionocedal.cl/detalle_contenido.php?vId=10\&vNam=Documentos\&vTip=0 Educação: Teoria e Prática/ Rio Claro/ Vol. 25, n.50/ p. XXX-XXX/ Set.-Dez. 2015.
} 
Figura 3 - Estructura Arzobispado de Santiago en Educación, La Pintana - Santiago de Chile

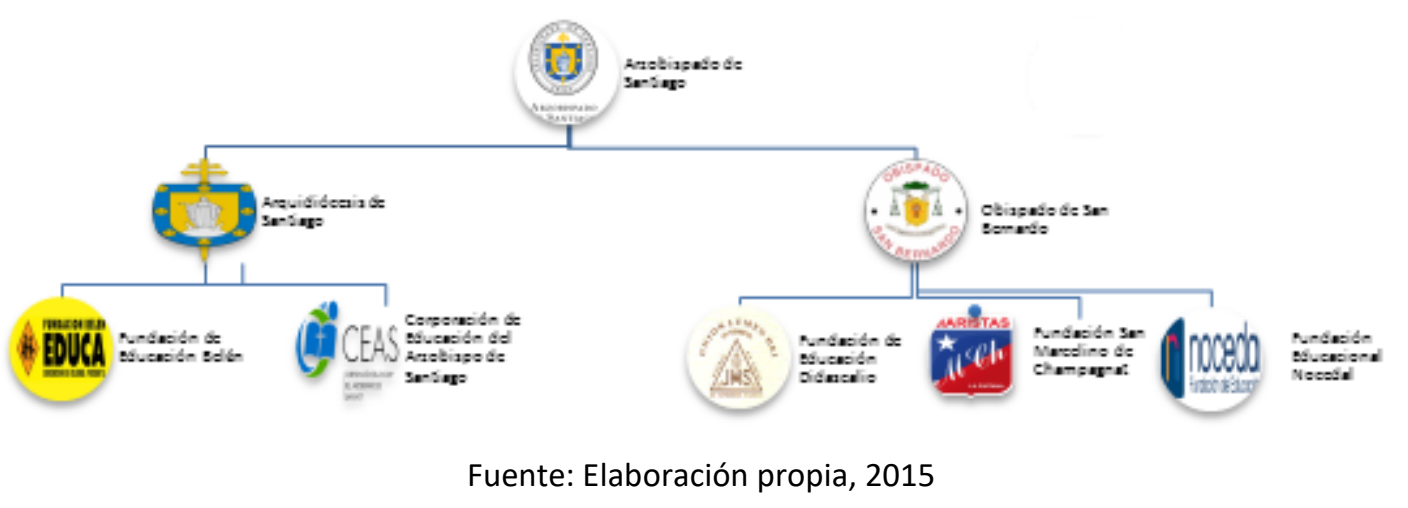

La Fundación Padre Álvaro Lavín pertenece al Hogar de Cristo, poderosa institución de beneficencia ligada a los jesuitas. Esta Fundación, ligada a cuatro colegios, recibió más de 50 millones de pesos (US 80.000 aprox.) vía de donaciones empresariales entre el 2008 y el 2009, además de desplegar diferentes campañas vinculadas con el Empresariado (Programa 1+1, Programa Done su Vuelto, Eventos y Voluntariados).

b) Grupos empresariales:son proveedores educativos, que sostienen colegios, mediante fundaciones que pertenecen a gremios empresariales o, que en su directorio, se encuentran miembros del mundo empresarial o se vinculan, mediante la recepción de donaciones y otro tipo de alianzas con grupos empresariales del área financiera y/o industrial.

Dentro de este grupo de sostenedores se constató la presencia de importantes redesconlos principales grupos industriales y financieros, a nivel nacional y transnacional. Por ejemplo, la Corporación Educacional Aprender, que tiene dos colegios, posee vínculos de colaboración con el Grupo Luksic (Banco de Chile, Compañía de Cervecerías Unidas S.A.), consorcio que posee la fortuna más grande del país, con presencia en diversos rubros (bancario, minero, alimenticio, medios de comunicación, empresas manufactureras y energéticas); el Grupo Banco Santander, transnacional bancaria con presencia en Latinoamérica y Europa; como también otras empresas privadas de diversa amplitud como Compañía de Seguros (Conosur), constructoras (Bio-Bio S.A.) y la Red interbancaria (Redbanc) (Figura 4). En su directorio también figura Mariana Aylwin y Sergio Molina, prominentes políticos democratacristianos, ex ministros de educación, consultores en temas de educación y férreos defensores de la educación privada. 
Figura 4 -Redes Comerciales y Empresariales de laCorporación Educacional Aprender

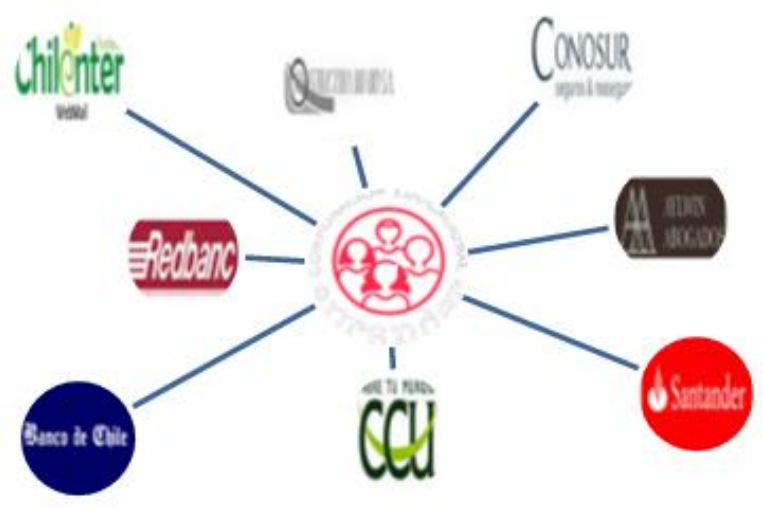

Fuente: Elaboración propia, 2015

La Sociedad de Instrucción Primaria (SIP), fundada en 1857, tiene diecisiete colegios en la Región Metropolitana, pertenece a la familia Matte Larraín o al llamado Grupo Matte, un conglomerado familiar de fuerte influencia en el país, que participa en distintos rubros económicos: forestal (Forestal Mininco - CMPC), eléctrico (Colbún), bancario (Bice), telecomunicaciones (Entel), construcción (Volcán S.A) y portuario (Puertos y Logística). Patricia Matte, líder de la SIP y miembro de los Legionarios de Cristo, ha sido asesora en educación del gobierno derechista de Sebastián Piñera y ha participado en distintas instancias de decisión sobre políticas educativas convocadas por los distintos bloques en el poder, como el Consejo Asesor Presidencial para la Educación, convocado el año 2006, y el Panel de Expertos, convocado el 2011, ambos en contexto de movilizaciones sociales por el derecho a la educación. La SIP se vincula directamente con la agencia de asesorías educativas Aptus Chile (Figura 5), que realiza asesorías técnicas en educación a diferentes sostenedores y establecimientos educacionales públicos y privados en gran parte del país, y con la Fundación CMPC, que, mediante alianzas público privadas, asesora a municipios en temas educativos y capacitación docente. 


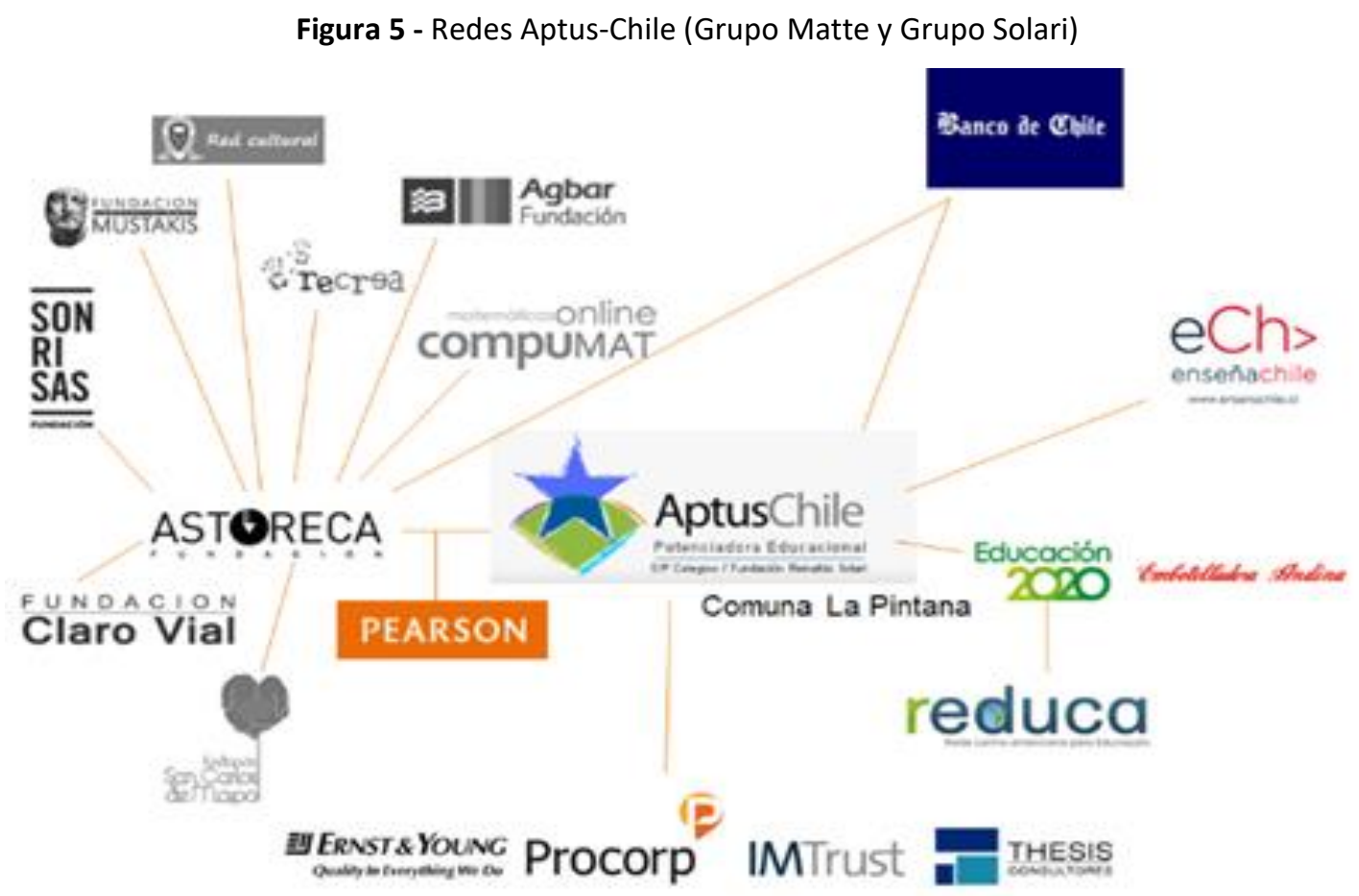

Fuente: Elaboración propia, 2015

Otro caso es el de la Corporación Educacional de la Construcción, perteneciente a la Cámara Chilena de la Construcción, la principal asociación gremial del área de la construcción. Posee ocho establecimientos técnico-profesionales en distintos puntos del país. La mayoría traspasados directamente por la dictadura, mediante el decreto 3166 de 1980, que entregó a distintos gremios el control de los mejores establecimientos técnicos del Estado. Entre el 2008 y el 2009 esta fundación recibió casi 80.000 dólares en donaciones vía ley 19.247 .

\subsection{Los grupos de poder en la Comuna de La Pintana: algunos datos}

Al mirar estos grupos en su conjunto, se pueden observar redes entre algunos de ellos, sean estos religiosos o empresariales. Por ejemplo la fundación Belén Educa, La Corporación Aprender, La Sociedad de Instrucción Primaria, La Fundación Nocedal, todos de la Pintana, se vinculan entre sí a través de una iniciativa conjunta, llamada Educando Juntos ${ }^{11}$, a la cual adscriben otros establecimientos, corporaciones y fundaciones de similares características que tiene escuelas en otras comunas de la región y en otras regiones del país. Otra supra red es la Fundación Irarrázaval Correa ligada una de las familias oligárquicas del país, la cual sin ser

\footnotetext{
${ }^{11}$ Ver portal web:http://www.educandojuntos.cl/dms/cat_770.html Educação: Teoria e Prática/ Rio Claro/ Vol. 25, n.50/ p. XXX-XXX/ Set.-Dez. 2015.
} 
una entidad sostenedora oficial ante el Ministerio de Educación se vincula, en calidad de apadrinamiento, con más de ciento de establecimientos en todo el país, entre ellos los establecimientos educativos de la Fundación Nocedal, la Fundación Aprender, la Fundación Marcelino Champagnat y la Fundación Educacional Belén, todas con presencia en la comuna de La Pintana. Estas redes interorganizacionales (BALL; CORBALÁN, 2013), constituyen redes entre ellos que coinciden en proyectos educativos, intercambio de recursos, patrocinadores. Su conexión semeja a un rizoma ${ }^{12}$ (Figura 6), sin un centro común, que hipotetizamos se extiende a través algunas comunas y regiones, de todo el país, constituyendo un proyecto educativo común, que es necesario hacer inteligible.

Figura 6 - Red grupos de poder en la comuna de La Pintana- Santiago Chile, 2014

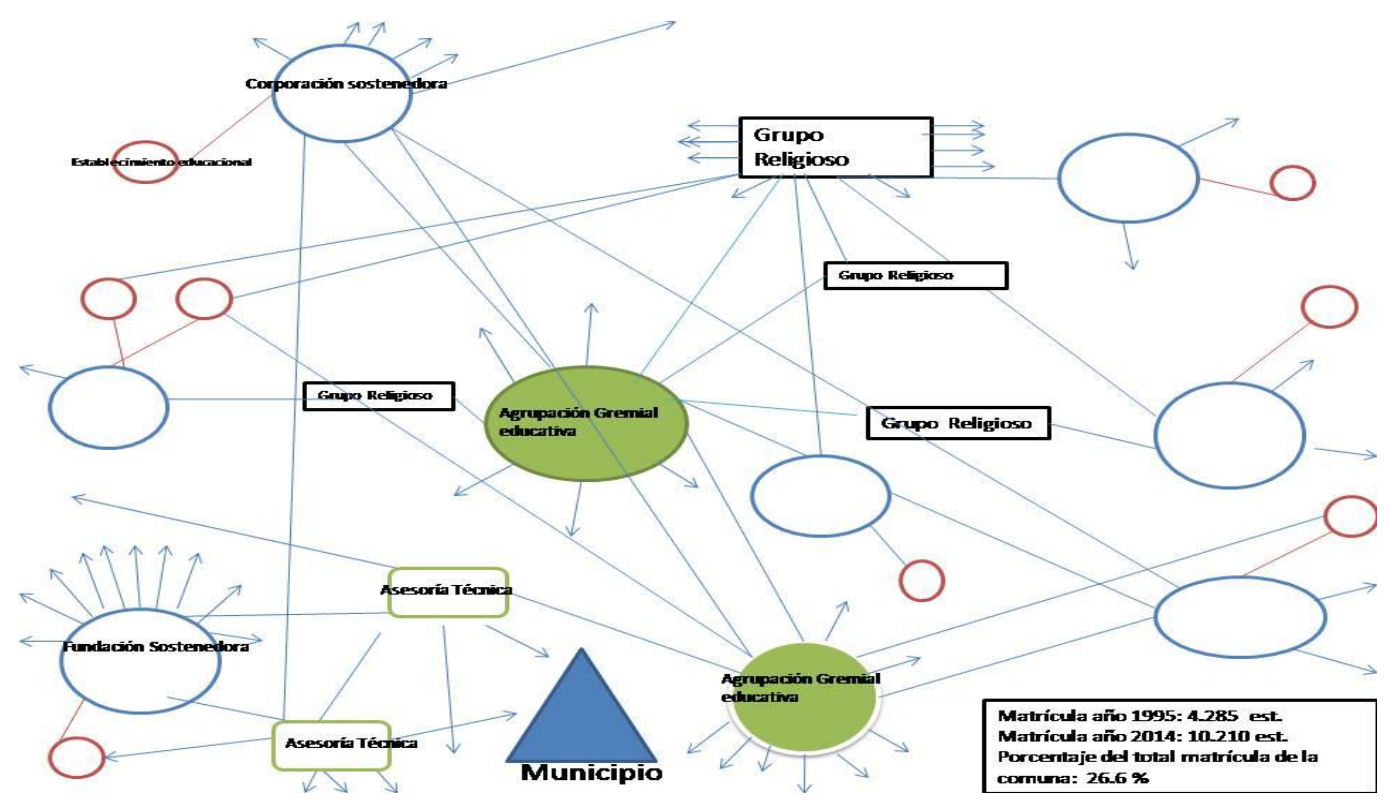

* Las flechas indican relaciones con instituciones sostenedoras y/o educativas de otras comunas y/o regiones del país. Fuente: elaboración propia, 2015

En consecuencia, si miramos este grupo como conjunto en la comuna de La Pintana, debemos señalar que, actualmente, posee una alta concentración de matrículas en sus establecimientos (Figura 7), alcanzando el 26\% aprox. de la matricula total de la comuna, situación que crece significativamente en la segunda mitad de la década de los 90’s y se consolida en la primera década del 2000 (Figura 8).

\footnotetext{
${ }^{12}$ Rizoma es una imagen o modelo descriptivo que se desprende de la botánica y designa un funcionamiento en red sin un centro común, ni jerarquía, que se extiende en determinado territorio o espacio.

Educação: Teoria e Prática/ Rio Claro/ Vol. 25, n.50/ p. XXX-XXX/ Set.-Dez. 2015.
} 
Figura 7 - Matrículas Poder Corporativo (1995-2014)

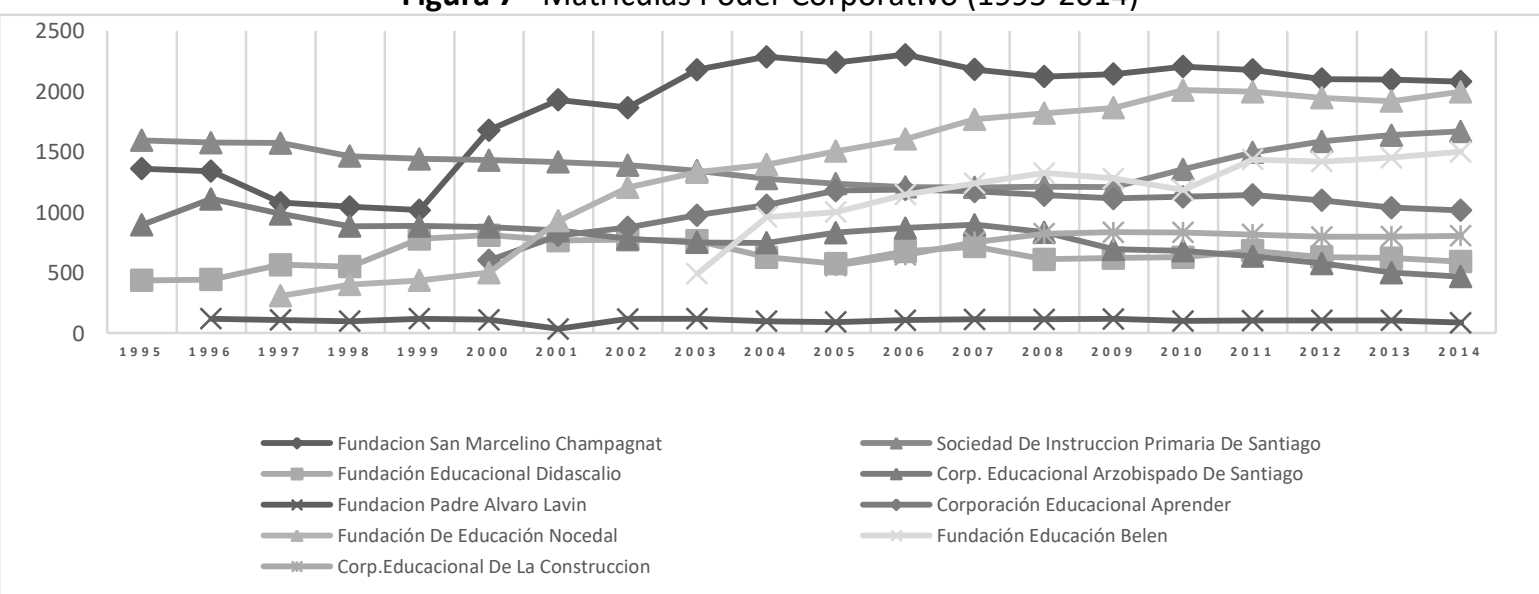

Fuente: Elaboración propia a partir de bases de datos del Ministerio de Educación (MINEDUC)

Figura 8 - Tendencia Matrículas Poder Corporativo

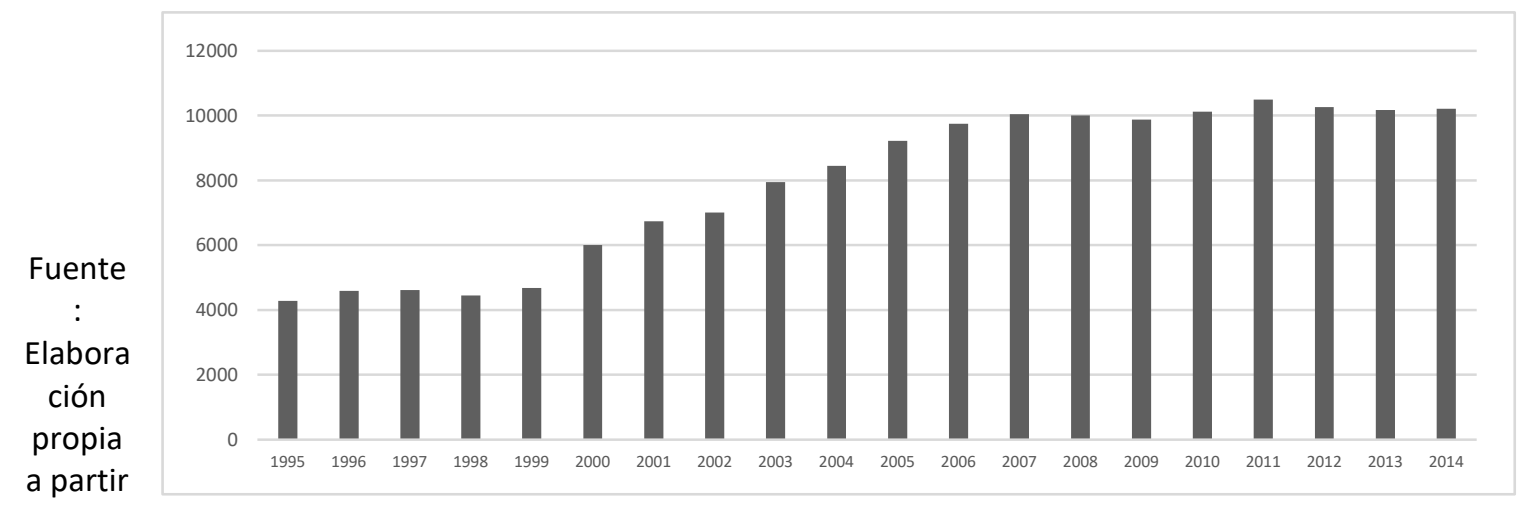

de bases de datos del Ministerio de Educación (MINEDUC)

Respecto a la presencia o ausencia de Financiamiento Compartido (FICOM) en los establecimientos que sostienen el Grupo de Poder, es posible señalar que el $50 \%$ los establecimientos educacionales utiliza FICOM, a diferencia de los Inversionistas poseen el porcentaje más alto (89\%), sin embargo aún sigue siendo un indicador alto respecto del resto de los sostenedores privados que no se encuentran en estas dos tipologías, 20\% (Figura 9).

Figura 9 -Porcentaje de Establecimientos que cobran FICOM según tipo de sostenedor

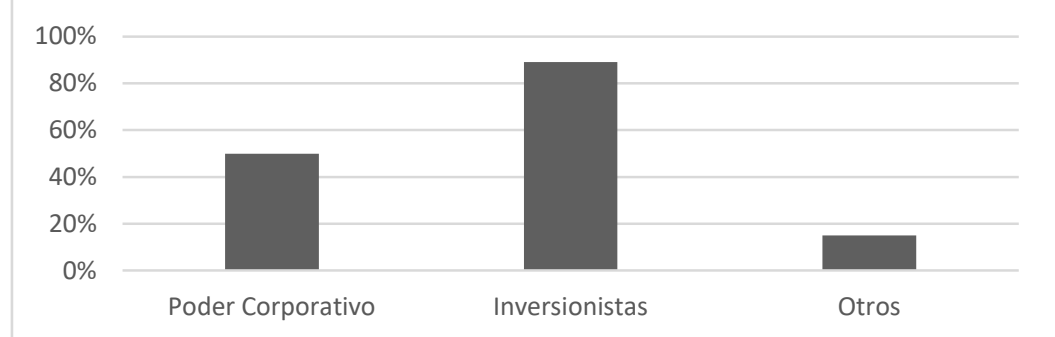

Fuente: Elaboración propia a partir de bases de datos del Ministerio de Educación (MINEDUC) 
A esto se agrega, el promedio del Índice de Vulnerabilidad Escolar (IVE) ${ }^{13}$ de los establecimientos de estos sostenedores, resultando uno de los más bajos de la comuna (junto con las escuelas de inversionistas). La población estudiantil más vulnerable no asiste a los establecimientos educacionales del Grupo de Poder (Figura 10). Esto permite hipotetizarque el cobro de mensualidad, en algunos casos, y alto nivel de selectividad de estos establecimientos, tiene un efecto segregador.

Figura 10 - Promedios Índice de Vulnerabilidad Escolar (IVE) según Tipo de Sostenedor

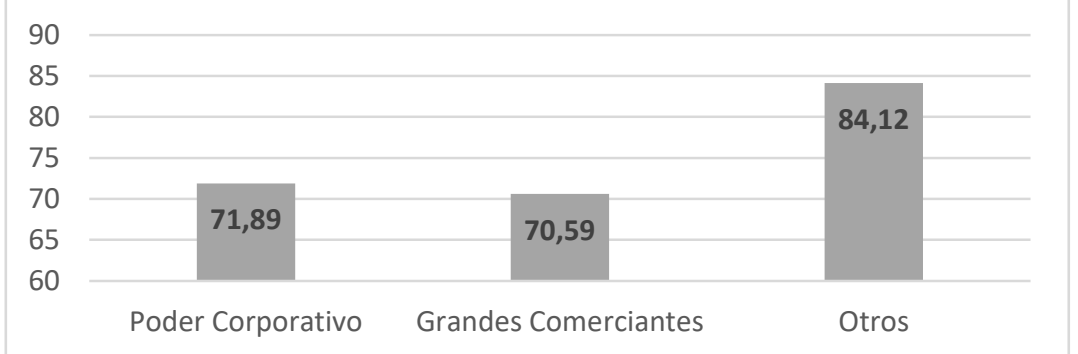

Fuente: Elaboración propia a partir de bases de datos del Ministerio de Educación (MINEDUC)

\section{Conclusiones}

En base a los resultados de la comuna de La Pintana, podemos señalar que hay una importante presencia de grupos de poder en la provisión educativa financiada por el Estado.Estos sostenedores tienen vínculos entre sí, con empresas, con escuelas, entidades sostenedoras, entidades de asesoras y con el propio Estado. Además, sus miembros han ocupado lugares de decisión en el diseño de las políticas educativas. Las redes que tienen entre ellos deben ser investigadas con detención, explorando sus vínculos regionales, nacionales y trasnacionales. También, debe estudiarse sus proyectos educativos y la influencia local que tienen, debido al alto nivel de selectividad y las zonas en que se encuentran estas intervenciones educativas. Esto último es relevante desde la perspectiva que la educación es un campo de relaciones de poder en disputa, y que hay por lo tanto resguardar lo democrático que sea nuestro sistema educativo.

La actual reforma educativa, en Chile, afecta escasamente estos grupos. La prohibición del lucro, el fin al financiamiento compartido y la eliminación de la selección no les afecta. En principio, estos grupos no lucran con la subvención estatal, todos reciben financiamiento

\footnotetext{
${ }^{13}$ El Índice de Vulnerabilidad Escolar (IVE) es un indicador que va de 0 a 100, mientras más alto el valor mayor vulnerabilidad, este índice asignado por la Junta Nacional de Auxilio Escolar y Becas, busca caracterizar la pobreza de la población escolar en las escuelas.

Educação: Teoria e Prática/ Rio Claro/ Vol. 25, n.50/ p. XXX-XXX/ Set.-Dez. 2015.
} 
complementario. Los establecimientos que cobran financiamiento compartido, ahora recibirán por parte del Estado una subvención por gratuidad. Respecto a la selección de estudiantes, la recién aprobada reforma elimina, gradualmente, los exámenes (en cinco años) y mantiene las entrevistas a las familias. Por lo tanto, está pendiente ver qué efecto tiene esto -de llevarse a cabo- en eliminar la segregación educativa, problema fundamental en un contexto en que grupos de poder económico y religiosos han alcanzado tanta influencia en la educación financiada por el Estado.

\section{Referencias}

ALMONACID, C. La Educación Particular Subvencionada como Cooperadora del Estado. En: BELlEI, C; CONTRERAS, D; VALENZUELA, J. (Eds.).La Agenda Pendiente en Educación. Profesores, Administradores y Recursos: Propuestas para la Nueva Arquitectura de la Educación Chilena. Santiago de Chile: Programa de Investigación en Educación, Universidad de Chile/UNICEF, 2008. p. 156-182.

ASSAÉL, J. et al. La empresa educativa chilena. Educ. Soc.,Campinas, v. 32, n. 115, p. 305 322, Abr./Jun. 2011.

BALL, S. Global Education INC. New Policy Networks and the Neo-liberal Imaginary. New York: Routledge, 2012.

BALL, S. Globalización, mercantilización y privatización: tendencias internacionales en Educación y Política Educativa. EducationPolicyAnalysis Archives, Buenos Aires, v. 22, n. 41, p. 1-13, Jun. 2014.

BALL, S.; CORBALÁN, F. Un nuevo escenario para la política y el negocio educativos. Cuadernos de Pedagogía, Barcelona, v.29, n. 435, p. 44-47, Jun. 2013.

BELLEI, C. Expansión de la educación privada y mejoramiento de la educación en Chile: evaluación a partir de la evidencia. Rev. Pensamiento Educativo, Santiago de Chile, v. 40, n. 1, p. 285-311, Abr. 2007.

CHILE. Ministerio de Desarrollo Social. Observatorio Social, Gobierno de Chile. Santiago: Reporte comunal/Comuna La Pintana, primer semestre, 2012.

CHILE. Instituto Nacional de Estadísticas.Censo de Población y Vivienda 2012.Santiago de Chile:INE, 2012.

CAMPAÑA LATINOAMERICANA DEL DERECHO A LA EDUCACIÓN - CLADE. Mapeo sobre tendencias de la privatización de la educación en América Latina y el Caribe. São Paulo:Campaña Latinoamericana por el Derecho a la Educación, 2015. (MIMEO) 
COLEGIO NOCEDAL. Proyecto educativo institucional Colegio Técnico Profesional Nocedal 2013. Disponible en:

http://www.colegionocedal.cl/detalle_contenido.php?vId=10\&vNam=Documentos\&vTip=0.

Extraído: 26 jul. 2014. 
CORBALÁN, F.; GONZÁLEZ J.; LIGÜEÑO, S. Identificación de la Propiedad y Dinámica de la Oferta Educativa Particular Subvencionada de la Región Metropolitana. Revista MAD, v.11, n. 20, p. 110-127. 2009.

CORNEJO, R. El Experimento Educativo Chileno 20 años después. Una mirada crítica a los Logros y Falencias del Sistema Escolar. REICE- Revista Electrónica Iberoamericana sobre Calidad, Eficacia y Cambio en Educación,Madrid, v. 4, n. 1, p.118-129, Ene. 2006

GONZÁLEZ, J.; LIGÜEÑO, S.; PARRA, D. El Proyecto Educativo del Complejo Religioso Empresarial y las Políticas de la Nueva Derecha en Educación. Revista Diatriba, n. 2, p. 3443, Ago.2012.

GONZÁLEZ, J. et al. Gubernamentalidad y provisión educativa privada en Chile. Reflexiones a partir de la identificación de la propiedad y dinámica de la oferta educativa privada en la Región Metropolitana. En: OYARZÚN, K. (Comp.).Sujetos y Actores Sociales:Reflexiones en el Chile de Hoy. Santiago: Universidad de Chile, 2010. p. 67-97.

INSTITUTO DE ESTUDIOS URBANOS UC. Indicador de Calidad de Vida Ciudades Chilenas (ICVU). Patrocinado por la Pontificia Universidad Católica y la Cámara Chilena de la Construcción. Santiago:Proyecto Anillos SOC1106, 2014. Disponible en: http://www.estudiosurbanos.uc.cl/images/investigaciones/Arturo_Orellana/ICVU/CChC_Pres entacion_ICVU_2014_FINAL_conferencia_Parte1.pdf. Extraído: 4 jun. 2014.

MORRIS, J. Las elites, los intelectuales y el consenso. Santiago de Chile: Pacífico, 1967.

REDONDO, J. El experimento chileno en educación. ¿Conduce a mayor equidad y calidad en educación?Última Década, Valparaíso, n. 22, p. 95-110, Ago.2005.

REDONDO, J. et al. Equidad y calidad de la educación en Chile. Santiago: Universidad de Chile, 2004.

SECRETARÍA DE PLANIFICACIÓN COMUNAL. Plan de Desarrollo Comunal (PLADECO) 2012-2016. Ilustre Municipalidad de La Pintana. Santiago de Chile: Municipalidad de La Pintana, 2012.

STAKE, R. Investigación con estudios de casos. Madrid: Ed. Morata, 1998.

VERGER, A. Nuevas Tendencias de privatización educativa. Las alianzas público-privadas en educación. En: FORO NACIONAL EDUCACIÓN DE CALIDAD PARA TODOS.Equidad y financiamiento de la educación. Análisis y debate en torno al financiamiento y realización del derecho a la educación. Cuaderno $n^{\circ} 8$, 1a. ed. Santiago de Chile, enero, 2013. p. 5-13: Disponible en:

http://educacionparatodos.cl/wp-content/uploads/2015/10/Cuaderno-del-Foro-Equidad-yFinanciamiento-de-la-Educaci\%C3\%83\%C2\%B3n.pdf. Extraído: 7 ene. 2014. 
Recebido em: 11/03/2015

Aprovado para publicação em: 24/08/2015

Publicado em: 22/12/2015

Educação: Teoria e Prática/ Rio Claro/ Vol. 25, n.50/ p.415-434/ Set.-Dez. 2015. 